\title{
ADSORCIÓN DE METALES PESADOS EMPLEANDO CARBONES ACTIVADOS PREPARADOS A PARTIR DE SEMILLAS DE AGUAJE
}

\author{
María del Rosario Sun-Kou ${ }^{* 1}$; Daniel Obregón-Valencia ${ }^{2}$; Ángela Pinedo-Flores ${ }^{1}$; Ana \\ Lucía Paredes-Doig'; Javier Aylas-Orejón ${ }^{1}$.
}

\begin{abstract}
RESUMEN
La semilla de aguaje (fruto típico de la selva amazónica) fue empleada como un material precursor para la preparación de carbón activado usando ácido fosfórico como agente activante en dos concentraciones diferentes: 0,75 y $1,0 \mathrm{~g}_{\mathrm{H} 3 \mathrm{PO} 4} / \mathrm{g}_{\text {Precursor. }}$ Las propiedades fisicoquímicas se estudiaron mediante mediciones de adsorción de nitrógeno, titulación Boehm, microscopía electrónica de barrido y espectroscopía de rayos $\mathrm{X}$ por energía dispersiva. Los carbones activados se utilizaron en la adsorción de tres metales pesados: plomo, cadmio y cromo. La máxima capacidad de adsorción se logró utilizando el carbón activado AG0,75_600 con soluciones con un $\mathrm{pH}$ mayor a 4; los valores obtenidos estaban en el siguiente orden: plomo $\left(74,8 \mathrm{mg} \mathrm{g}^{-1}\right)$, cadmio $\left(26,5 \mathrm{mg} \mathrm{g}^{-1}\right)$, cromo $\left(18,8 \mathrm{mg} \mathrm{g}^{-1}\right)$. Los factores más importantes que influyen en el proceso de adsorción fueron el $\mathrm{pH}$ de la solución, la estructura porosa y la acidez superficial del carbón activado.
\end{abstract}

Palabras clave: carbón activado, semillas de aguaje, plomo, cadmio, cromo.

\section{ADSORPTION OF HEAVY METALS USING ACTIVATED CARBONS PREPARED FROM AGUAJE STONES}

\begin{abstract}
Aguaje seed (a typical Amazonian forest fruit) was used as a precursor material for the production of activated carbon using phosphoric acid as activating agent in two different concentrations: 0,75 and $1,0 \mathrm{~g}_{\mathrm{H} 3 \mathrm{PO} 4} / \mathrm{g}_{\text {Precursor }}$. The physicochemical properties were studied by $\mathrm{N}_{2}$ sorption measurements, Boehm titration, scanning electron microscopy and energydispersive X-ray spectroscopy. Activated carbons were used in the adsorption of three heavy metals: lead, cadmium and chromium. The maximum adsorption capacity was achieved using activated carbon AG0,75_600 in a solution with a $\mathrm{pH}$ greater than 4 and the values obtained were in the following order: lead $\left(74,8 \mathrm{mg} \mathrm{g}^{-1}\right)$, cadmium $\left(26,5 \mathrm{mg} \mathrm{g}^{-1}\right)$, chromium $\left(18,8 \mathrm{mg} \mathrm{g}^{-1}\right)$. The most important factors influencing the adsorption process were the $\mathrm{pH}$ of the solution, the porous structure and surface acidity of the activated carbon.

Key words: activated carbon, aguaje stones, lead, cadmium, chromium.

\section{INTRODUCCIÓN}

El agua es un recurso esencial para la vida, pero debido a la irresponsabilidad de las personas y a la ausencia de regulaciones gubernamentales que controlen la emisión de los efluentes

\footnotetext{
Sección Química-Departamento de Ciencias, PUCP. Miguel-Lima-Perú

Teléfono:(51)626-2000-Fax:(51)626-2853-*Email:msun@pucp.edu.pe
}

2 Instituto de Corrosión y Protección - Pontificia Universidad Católica del Perú - CEP: Lima 32 - San
\end{abstract}


domésticos e industriales, las diversas fuentes de aguas se encuentran en muchos casos contaminadas con sustancias orgánicas y metales pesados altamente tóxicos, que afecta tanto al ecosistema como a la salud de los pobladores que viven en las riberas de los cursos de agua. Entre los metales pesados con mayor toxicidad e incidencia ${ }^{1}$ se encuentran el plomo, el cadmio y el cromo, cuyos límites máximos permisibles ${ }^{2}$ (MLP) son: $0,05 \mathrm{mg} / \mathrm{L}$ para el plomo y cromo, y $0,003 \mathrm{mg} / \mathrm{L}$ para el cadmio según la legislación peruana.

Entre los procesos aplicados al tratamiento del agua están: precipitación, ósmosis reversa, reacciones de oxidación avanzada, intercambio iónico, adsorción, entre otros. Siendo el proceso de adsorción el más recomendado por su versatilidad y bajo costo.

El carbón activado (CA) es el adsorbente más usado en la industria. El CA tradicionalmente se obtiene a partir del carbón mineral, el cual es un recurso no renovable; esto conduce, además, a que se incremente el precio. Los materiales lignocelulósicos (residuos agrícolas) son una fuente renovable, abundante y económica para la preparación de carbones activados. Bajo este contexto, en este trabajo se ha utilizado la semilla de aguaje (fruto típico de la amazonía) como material precursor del carbón activado. Para la preparación del CA se siguió una metodología probada en trabajos previos ${ }^{3-4}$ en donde se encontró que los CA que presentaron una mayor capacidad de adsorción de metales pesados en solución acuosa, fueron obtenidos a una temperatura de activación de $600^{\circ} \mathrm{C}$. El objetivo de este trabajo es estudiar la capacidad de adsorción de los iones $\mathrm{Pb}^{2+}, \mathrm{Cr}_{\text {(Total }}, \mathrm{Cd}^{2+}$ a diferentes $\mathrm{pH}$ empleando $\mathrm{CA}$ preparados a partir de la semilla de aguaje, con diferentes razones de impregnación y activados a la temperatura de $600^{\circ} \mathrm{C}$.

\section{PARTE EXPERIMENTAL}

Las semillas de aguaje empleadas como material precursor del CA, fueron recolectadas en el Departamento de Ucayali - Perú.

\section{Preparación de carbón activado:}

El material precursor (50 g) fue mezclado con una concentración determinada de ácido fosfórico $\left(\mathrm{H}_{3} \mathrm{PO}_{4}\right.$, Merck $)$ a fin de obtener dos razones de impregnación 0,75 y 1,0 $\mathrm{g}_{\mathrm{H} 3 \mathrm{PO} 4} / \mathrm{g}_{\mathrm{Precursor}}$. La mezcla fue filtrada al vacío y colocada en la estufa a $85^{\circ} \mathrm{C}$ por $24 \mathrm{~h}$. El material impregnado y seco fue colocado en el interior de un reactor tubular. Dicho reactor se colocó dentro de un horno y se calentó hasta una temperatura de $600{ }^{\circ} \mathrm{C}$ durante una hora. Dentro del reactor se mantuvo un flujo constante de $\mathrm{N}_{2}$ de $100 \mathrm{~cm}^{3} / \mathrm{min}$. La nomenclatura empleada en este estudio fue: $\mathrm{AG}=$ semilla de aguaje, seguido de los valores del grado de impregnación y la temperatura activación. Por ejemplo, AG0,75_600 fue preparado con una razón de impregnación de $0,75 \mathrm{~g}_{\mathrm{H} 3 \mathrm{PO} 4} / \mathrm{g}_{\text {Precursor }} \mathrm{y}$ a una temperatura de activación de $600{ }^{\circ} \mathrm{C}$.

\section{Caracterización del carbón activado}

Se utilizó las siguientes técnicas:

Titulación Boehm: Se pesó 0,25 mg de CA y se mezcló con $50 \mathrm{~mL}$ de una solución de $\mathrm{NaOH}$ $0,1 \mathrm{~N}$ estándar, bajo agitación durante $24 \mathrm{~h}$ a temperatura ambiente. Concluido dicho tiempo, la mezcla fue separada por filtración y se tituló la solución con $\mathrm{HCl} 0,1 \mathrm{~N}$ estándar para cuantificar la acidez superficial del CA.

Adsorción de nitrógeno: Se empleó un equipo Micromeritics GEMINI VII, modelo 2390t. Previo al análisis, las muestras fueron desgasificadas en vacío a una temperatura de $250{ }^{\circ} \mathrm{C}$ durante $2 \mathrm{~h}$. Se cuantificó la cantidad de nitrógeno gaseoso retenida en la superficie del CA a $77 \mathrm{~K}$ hasta presiones relativas cercanas a 1. La superficie específica fue calculada de acuerdo al método BET. Se determinó el área microporosa empleando el método t-plot. El área 
mesoporosa se calculó por diferencia entre la superficie específica $\left(\mathrm{S}_{\mathrm{BET}}\right)$ y el área microporosa. El diámetro medio de poro se determinó empleando el método BJH.

Microscopía electrónica de barrido (SEM): Se empleó un equipo Phillips modelo 505DX. Las muestras fueron metalizadas previamente con una fina capa de oro a fin de hacer las muestras conductoras.

Espectrometría de rayos X por energía dispersiva (EDX): Se utilizó un equipo FEI modelo QUANTA 200 con un sistema de análisis integrado OXFORD INSTRUMENTS AnalyticalInca con detectores de espectrometría de rayos X por energía dispersiva, se aplicó un voltaje de aceleración: $0,2-30 \mathrm{kV}$ y distancia de foco WD: $10 \mathrm{~mm}$.

\section{Proceso de adsorción}

Mediante los ensayos cinéticos realizados previamente ${ }^{3-4}$ se determinó que el tiempo de equilibrio para la adsorción de los iones de $\mathrm{Pb}^{2+}, \mathrm{Cd}^{2+}$ y $\mathrm{Cr}_{\text {(Total) }}$ fue de aproximadamente $2 \mathrm{~h}$. Para asegurar haber alcanzado las condiciones de equilibrio, en este trabajo, todas las isotermas se realizaron con un tiempo de contacto de $4 \mathrm{~h}$.

Los ensayos de adsorción se llevaron a cabo a temperatura ambiente con concentraciones iniciales del adsorbato entre 2-80 ppm para cada metal. Otro parámetro que se estudió fue el $\mathrm{pH}$ de la solución metálica que varió entre 2-5. Para esto se colocó en diferentes viales $15 \mathrm{mg}$ de CA con $20 \mathrm{~mL}$ de las soluciones preparadas a distintas concentraciones del metal. La capacidad de adsorción $\left(\mathrm{q}_{\mathrm{e}}\right)$ que indica la cantidad de metal adsorbido en el equilibrio fue calculado de acuerdo a la ecuación siguiente:

$$
q_{e}=\frac{\left(c_{0}-c_{e}\right) V}{W}
$$

Ecuación 1

donde, $\mathrm{C}_{\mathrm{o}}$ and $\mathrm{C}_{\mathrm{e}}\left(\mathrm{mg} . \mathrm{L}^{-1}\right)$ son la concentración inicial y en el equilibrio de cada metal, $\mathrm{V}(\mathrm{L})$ es el volumen de la fase acuosa; y W (g) es el peso del CA. La concentración residual de los metales fue analizada usando un equipo de absorción atómica Perkin Elmer modelo 3110. Los resultados experimentales fueron correlacionados con los siguientes modelos de isoterma:

a) Modelo de Langmuir: este modelo asume que la adsorción ocurre en un número finito y definido de sitios localizados idénticos y equivalentes energéticamente que forman una monocapa. Esto ocurre sin ninguna interacción lateral o impedimento estérico entre las moléculas adsorbidas. La forma lineal del modelo de Langmuir se representa mediante la ecuación:

$$
\frac{C_{e}}{q_{e}}=\frac{1}{K_{L} q_{m a ́ x}}+\frac{C_{e}}{q_{\text {máx }}}
$$

Ecuación 2

donde, $\mathrm{q}_{\mathrm{e}}\left(\mathrm{mg} \cdot \mathrm{g}^{-1}\right)$ representa la capacidad de adsorción o cantidad del adsorbato retenido en el adsorbente en el equilibrio; $\mathrm{C}_{\mathrm{e}}\left(\mathrm{mg} \cdot \mathrm{L}^{-1}\right)$, es la concentración del adsorbato presente en la solución en el equilibrio; $\mathrm{q}_{\text {máx }}\left(\mathrm{mg} \cdot \mathrm{g}^{-1}\right)$, es la capacidad máxima de adsorción $\mathrm{y}, \mathrm{K}_{\mathrm{L}}\left(\mathrm{L}_{\mathrm{mg}} \mathrm{m}^{-1}\right)$, representa la constante de Langmuir relacionada con el calor de adsorción.

b) Modelo de Freundlich: este modelo describe una adsorción no ideal, reversible y heterogénea. Se puede aplicar este modelo para una adsorción en multicapa sin una distribución uniforme de energía o afinidad de adsorción sobre una superficie heterogénea. El modelo en su forma lineal se representa por: 


$$
\log q_{e}=\log K_{F}+\frac{1}{n} \log C_{e}
$$

Ecuación 3

donde, $\mathrm{q}_{\mathrm{e}}\left(\mathrm{mg} \cdot \mathrm{g}^{-1}\right)$ es la capacidad de adsorción en el equilibrio y $\mathrm{C}_{\mathrm{e}}\left(\mathrm{mg} \cdot \mathrm{L}^{-1}\right)$ es la concentración de soluto presente en la solución en el equilibrio. La constante $\mathrm{K}_{\mathrm{F}}\left(\mathrm{mg}^{1-1 / n} \mathrm{~L}^{1 / n} \mathrm{~g}^{-1}\right)$ es un parámetro de la capacidad de adsorción del material y $1 / \mathrm{n}$ (adimensional) representa una medida de la intensidad de la adsorción. El tipo de isoterma depende del valor 1/n; si dicho valor es mayor o igual a 0,5 la curva de la isoterma es cóncava hacia arriba y la adsorción es desfavorable.

c) Modelo de Redlich-Peterson: Este modelo, debido a su versatilidad, permite representar un sistema de adsorción en equilibrio para un amplio rango de concentraciones y puede ser aplicado tanto para sistemas homogéneos como heterogéneos. La ecuación lineal de este modelo se representa por:

$$
\ln \left(A_{R P} \frac{C_{e}}{q_{e}}-1\right)=G \ln \left(C_{e}\right)+\ln \left(B_{R P}\right)
$$

Ecuación 4

donde, $\mathrm{q}_{\mathrm{e}}\left(\mathrm{mg} \cdot \mathrm{g}^{-1}\right)$ es la capacidad de adsorción en el equilibrio, $\mathrm{C}_{\mathrm{e}}\left(\mathrm{mg} \cdot \mathrm{L}^{-1}\right)$ es la concentración de soluto presente en la solución en el equilibrio; $\mathrm{A}_{\mathrm{RP}}, \mathrm{B}_{\mathrm{RP}} \mathrm{y} \mathrm{G}$ son los parámetros de RedlichPeterson.

El valor de $G$ varía entre 0 y 1 y determina el grado de heterogeneidad de la superficie, siendo más homogéneo al acercarse a 1 . De esta manera, la constante $G$ puede caracterizar una isoterma: si G es cercano a 1, la isoterma se aproxima más a la de Langmuir, mientras que si G es cercano a 0 , la isoterma se aproxima a la de Freundlich.

d) Modelo de Temkin: Este modelo asume que hay una disminución del valor de adsorción lineal en lugar de logarítmica, como en el modelo de Freundlich, se expresa como:

$$
q_{e}=\frac{R T}{b}\left(\ln A_{T}\right)+\left(\frac{R T}{b}\right) \ln C_{e}
$$

Ecuación 5

Donde, $\mathrm{RT} / \mathrm{b}$ se relaciona con el calor de adsorción, $\mathrm{R}\left(\mathrm{Jmol}^{-1} \mathrm{~K}^{-1}\right)$, es la constante universal de los gases, $\mathrm{T}(\mathrm{K})$ es la temperatura, $\mathrm{b}\left(\mathrm{J} \cdot \mathrm{mol}^{-1}\right)$ es la variación de la energía de adsorción y $\mathrm{A}_{\mathrm{T}}$ $\left(\mathrm{L} \cdot \mathrm{mg}^{-1}\right)$ es la constante del enlace en el equilibrio correspondiente a la máxima energía de enlace.

La desviación estándar normalizada entre los valores experimentales y los valores predichos por los modelos fue empleada para evaluar el modelo que presentaba el mejor ajuste. La desviación estándar normalizada fue calculada de la siguiente manera:

$$
\Delta \mathrm{q}_{\mathrm{e}}(\%)=100 \sqrt{\frac{\sum[q e(\exp )-q e(p r o) / q e(\exp )]^{2}}{N}}
$$

Ecuación 6 
Donde, $\mathrm{q}_{\mathrm{e}(\exp )}$ es el valor experimental, $\mathrm{q}_{\mathrm{e}(\text { pro })}$ es el valor correspondiente $\mathrm{al} \mathrm{q}_{\mathrm{e}}$ pronosticado de acuerdo a la ecuación bajo estudio, $\mathrm{N}$ es el número de mediciones. En este estudio el menor valor obtenido de desviación estándar normalizado indicó el mejor ajuste de los resultados experimentales.

\section{RESULTADOS Y DISCUSIÓN}

La figura 1-izquierda representa las isotermas de adsorción de $\mathrm{N}_{2}$ y la figura 1-derecha muestra la distribución de poros de los carbones activados preparados con distintos grados de impregnación. Se puede apreciar que todas las isotermas son del Tipo IV lo cual es característico de materiales micro y mesoporosos. Al aumentar el grado de impregnación se observa un incremento de la superficie específica $\left(\mathrm{S}_{\mathrm{BET}}\right)$ con una reducción de la mesoporosidad (tabla 1). Asimismo, se observa una distribución de poros más heterogénea para el caso de la muestra AG0,75_600 (figura 1- derecha), lo cual se evidencia en la mayor área mesoporosa obtenida (tabla 1). En ambos CA el diámetro promedio de poro se mantuvo constante $(2,6 \mathrm{~nm})$.

La acidez superficial disminuye con el incremento de la razón de impregnación (tabla 1). Este comportamiento puede explicarse considerando que para un mayor grado de impregnación se genera una menor cantidad de grupos oxigenados lo cual fue comprobado con los resultados de EDX (figura 2). Este comportamiento concuerda con los resultados reportados por Puziy et $\mathrm{al}^{5}$.
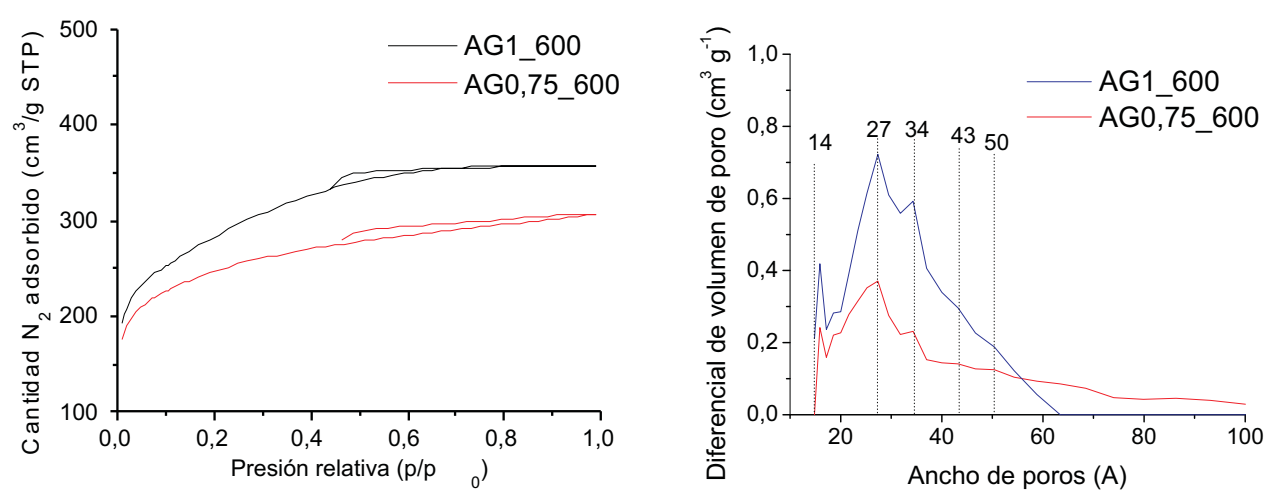

Figura 1. Isotermas de adsorción de $\mathrm{N}_{2} \mathrm{y}$ distribución de poros de las muestras AG1_600 AG0,75_600.

Tabla 1. Caracterización textural y de acidez superficial de AG0,75_600 y AG1_600

\begin{tabular}{|c|c|c|c|c|c|}
\hline \multirow{2}{*}{ Muestra } & \multicolumn{3}{|c|}{ Área $\left(m^{2} g^{-1}\right)$} & \multirow{2}{*}{$\begin{array}{l}\text { Poro: } d_{\text {promedio }} \\
(\mathrm{nm})\end{array}$} & \multirow{2}{*}{$\begin{array}{c}\text { Acidez superficial }\left(\mathrm{mmol}^{+}\right. \\
\left.\qquad \mathrm{g}^{-1}\right)\end{array}$} \\
\hline & $\mathbf{S}_{\text {BET }}$ & $\mathbf{S}_{\text {Meso }}$ & SMicro $_{\text {M }}$ & & \\
\hline AG0,75_600 & 905 & 140 & 765 & 2,6 & 2,43 \\
\hline AG1_600 & 1014 & 76 & 937 & 2,6 & 2,29 \\
\hline
\end{tabular}






Figura 2. Análisis EDX de las muestras AG0,75_600 y AG1_600.

En la Figura 3 se observan las micrografías SEM de AG0,75_600 y AG1_600 a una magnificación entre 400X y 1000X. La muestra AG0,75_600 evidencia una mayor porosidad del material (figura 3: AG0,75_600-400X), con cavidades que presentan en su interior poros de un diámetro aproximado $8 \mu \mathrm{m}$ en comparación con el otro carbón AG1_600.



AG1_600-400X

AG0,75_600-400X

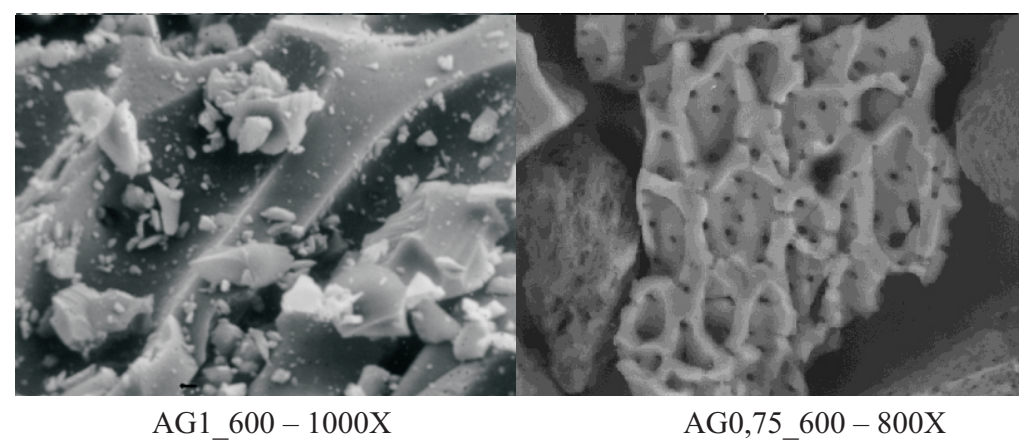

Figura 3. Micrografías SEM de AG1_600 (lado izquierdo) y AG0,75_600 (lado derecho) con aumentos entre 400X y 1000X. 
En trabajos previos ${ }^{3-4}$ se realizó los ensayos cinéticos a partir de las cuales se determinó que la adsorción de los metales requería un tiempo de $2 \mathrm{~h}$ para llegar al equilibrio. En este trabajo se empleó un tiempo de contacto de $4 \mathrm{~h}$ para asegurar que el sistema siempre alcanzara el equilibrio.

La forma de la curva cinética (resultados no mostrados) indicó un ascenso lento hasta alcanzar las condiciones de equilibrio. Las curvas cinéticas obtenidas se ajustaron al modelo de pseudo segundo orden lo que permitió elucidar que el proceso de adsorción es el resultado de una interacción preferentemente del tipo químico (quimisorción).En la figura 4 se muestra las isotermas de adsorción de los tres metales. Las isotermas de cadmio y cromo a pH 2 son cóncavas y se asemejan a una curva tipo L según la clasificación de Giles ${ }^{7}$, mientras que a pH 5 las isotermas son del tipo $\mathrm{H}$ (presenta una pendiente inicial muy alta), la cual se caracteriza por una fuerte adsorción del adsorbato. En estas isotermas se alcanza la condición estacionaria a valores de concentraciones en el equilibrio $\left(\mathrm{C}_{\mathrm{e}}\right)$ de aproximadamente $20 \mathrm{mg} \mathrm{L}^{-1}$ a pH $=2$; pero al variar el pH a 5 se incrementa el valor $\mathrm{de}_{\mathrm{e}}$ (figura 4 ). Con relación al plomo, se observa que las isotermas son del tipo $\mathrm{H}$ (independiente del $\mathrm{pH}$ ) y no alcanza una condición estacionaria a medida que se incrementa los valores de Ce. La capacidad de adsorción aumenta con el incremento del pH 2 a 5 . Estos resultados se corroboran con otras investigaciones ${ }^{8-9}$ realizadas en que se observó que la adsorción del ion de plomo fue superior a la del cadmio. De acuerdo a El-Hendawy ${ }^{10}$, el ion de plomo se adsorbe en mayor cantidad en comparación que el ion de cadmio debido a su baja solubilidad y a su alto grado de acomplejamiento el cual se relaciona con la interacción existente entre el metal y los grupos funcionales de la superficie del carbón.

Se conoce que el CA presenta en su superficie una serie de grupos funcionales de los cuales algunos son de carácter ácido y otros básicos; esto le confiere al carbón su naturaleza anfótera. En un estudio realizado por Pandey et al $^{11}$, se comparó los grupos funcionales superficiales del CA con la de otra sustancia como el ácido húmico; los investigadores observaron que se puede formar compuestos de coordinación entre el ácido húmico y los metales. La estabilidad reportada para los complejos metal-ácido húmico fue la siguiente:

$$
\mathrm{Cu}>\mathrm{Fe}>\mathrm{Pb}>\mathrm{Ni}>\mathrm{Co}>\mathrm{Ca}>\mathrm{Cd}>\mathrm{Zn}>\mathrm{Mn}>\mathrm{Mg} \text {. }
$$

El comportamiento descrito anteriormente puede estar asociado a la naturaleza del ion en estudio. En el caso del ion de plomo, el cual es considerado un ácido blando ${ }^{12}$, esto significa que la adsorción del plomo no es afectada por el pH de la solución o la acidez superficial del CA. Como se aprecia en la figura 4, las isotermas de plomo presentan para el mismo CA, un comportamiento similar a distinto $\mathrm{pH}$. En el caso del cadmio y el cromo, estos son considerados ácidos duros ${ }^{12}$, lo cual indicaría que son sensibles al $\mathrm{pH}$ de la solución y a la acidez de la superficie del CA.

Teniendo en cuenta que el punto isoeléctrico $\left(\mathrm{pH}_{\mathrm{PZC}}\right)$ de la muestra AG0,75_600 es de 2,55 (evaluado en un trabajo anterior), esto nos indicaría que en soluciones acuosas a pH menores al punto isoeléctrico la superficie del CA se encuentra cargada en forma positiva lo cual crearía repulsiones electroestáticas con los iones $\mathrm{Cd}^{2+} \mathrm{y} \mathrm{Cr}_{\left(\mathrm{Total}_{)}\right.}$; además, a pH bajos se presenta un efecto competitivo en la solución entre los iones $\mathrm{H}^{+}$y los iones metálicos, lo cual contribuye a una reducción de la capacidad de adsorción de los metales. Por el contrario, a pH 5 que es mayor al $\mathrm{pH}_{\mathrm{PZC}}$, la superficie del $\mathrm{CA}$ se encontraría cargada en forma negativa lo cual favorecería las atracciones electrostáticas con los iones metálicos $\mathrm{Cd}^{2+} \mathrm{y} \mathrm{Cr}_{\text {(Total) }}$. Asimismo, se puede observar que la adsorción de cadmio y cromo es mayor en el carbón AG0,75_600. Esto 
se puede explicar debido a la mayor acidez superficial de dicho carbón. Adicionalmente, entre estos iones metálicos, el cromo es considerado un ácido más duro que el cadmio, por lo que su adsorción es menos favorecida (figuras 4-B1 y 4-B2).
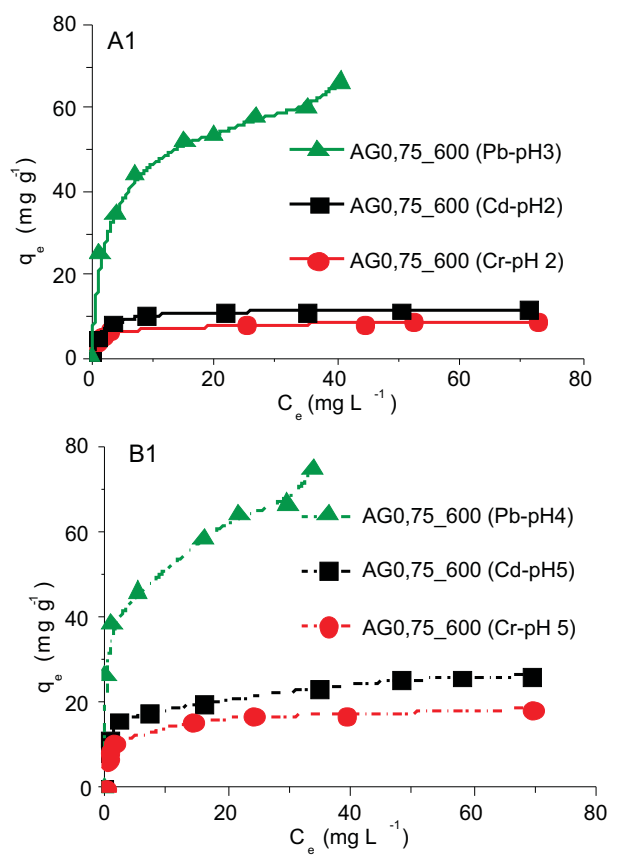
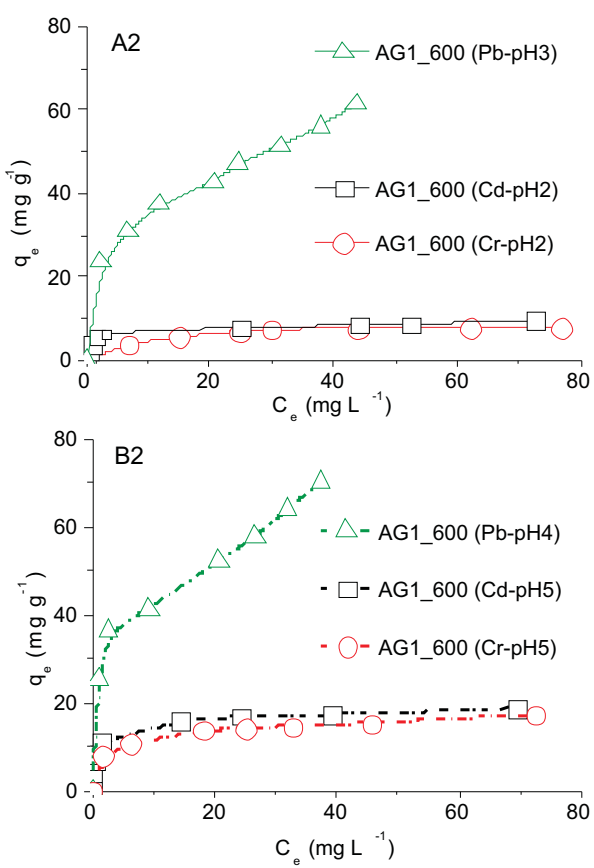

Figura 4. Isotermas de adsorción de plomo, cadmio y cromo en soluciones con distintos pH utilizando los carbones activados AG0,75_600 y AG1_600.

A partir de las isotermas de la figura 4 se observa el siguiente orden de adsorción sobre el carbón activado: $\mathrm{Pb}$ (II) > Cd (II) > Cr (III). Esto concuerda con el tamaño relativo de los respectivos iones solvatados. El Cr (III), por su mayor carga y menor radio, posee una mayor capacidad de hidratación y, por ende, un mayor radio solvatado, lo que dificulta su retención en la superficie de carbón activado y explica su menor adsorción en relación con los otros dos metales. Por otro lado, comparando ambos iones divalentes, el $\mathrm{Pb}$ (II), de mayor tamaño como ion libre posee un menor radio solvatado que el Cd(II) y encabeza el orden de adsorción.

Los valores experimentales de las isotermas obtenidas a diferentes $\mathrm{pH}$ se correlacionaron con los modelos de isotermas de adsorción de: Langmuir, Freundlich, Temkin y Redlich-Peterson. Los parámetros correspondientes a dichos modelos se muestran en las tablas 2 y 3 . 
Tabla 2. Parámetros de las isotermas obtenidas de la adsorción de metales pesados empleando los carbones activados AG0,75_600 y AG1_600.

\begin{tabular}{|c|c|c|c|c|c|c|}
\hline \multirow[b]{2}{*}{ Muestra } & \multicolumn{2}{|c|}{$\mathrm{Pb}(\mathrm{pH} 3)$} & \multicolumn{2}{|c|}{$\mathrm{Cr}(\mathrm{pH} 2)$} & \multicolumn{2}{|c|}{ Cd (pH 2) } \\
\hline & $\mathbf{0 , 7 5}$ & 1 & 0.75 & 1 & $\mathbf{0 , 7 5}$ & 1 \\
\hline \multicolumn{7}{|c|}{ Freundlich } \\
\hline $\mathrm{K}_{\mathrm{F}}$ & 23,91 & 17,02 & 4,89 & 2,67 & 6,08 & 3,83 \\
\hline $1 / \mathrm{n}$ & 0,28 & 0,30 & 0,15 & 0,27 & 0,18 & 0,23 \\
\hline$r^{2}$ & 0,98 & 0,99 & 0,91 & 0,88 & 0,81 & 0,80 \\
\hline$\Delta \mathrm{q}(\%)$ & 4,66 & 7,02 & 8,33 & 8,04 & 12,33 & 24,40 \\
\hline \multicolumn{7}{|l|}{ Langmuir } \\
\hline Qm & 68,41 & 68,28 & 9,00 & 8,77 & 11,87 & 9,47 \\
\hline $\mathrm{K}_{\mathrm{L}}$ & 0,25 & 0,12 & 0,67 & 0,15 & 0,74 & 0,48 \\
\hline $\mathrm{r}^{2}$ & 0,99 & 0,96 & 1,00 & 1,00 & 1,00 & 0,99 \\
\hline$\Delta \mathrm{q}_{\mathrm{e}}(\%)$ & 11,64 & 14,55 & 11,25 & 4,62 & 1,66 & 18,98 \\
\hline \multicolumn{7}{|l|}{ Temkin } \\
\hline B & 0,21 & 0,19 & 2,50 & 1,48 & 1,67 & 1,98 \\
\hline $\mathrm{A}_{\mathrm{T}}$ & 5,96 & 2,02 & 153,79 & 2,28 & 69,79 & 31,73 \\
\hline$r^{2}$ & 0,99 & 0,93 & 0,96 & 0,91 & 0,87 & 0,93 \\
\hline$\Delta \mathrm{q}_{\mathrm{e}}(\%)$ & 3,85 & 8,25 & 7,01 & 6,37 & 10,64 & 21,01 \\
\hline \multicolumn{7}{|c|}{ Redlich-Peterson } \\
\hline $\mathrm{G}$ & 0,84 & 0,80 & 1,00 & 0,82 & 0,99 & 0,97 \\
\hline$A_{R P}$ & 50 & 28 & 9,2 & 2,50 & 9 & 8 \\
\hline $\mathrm{B}_{\mathrm{RP}}$ & 1,38 & 1,03 & 1,04 & 0,61 & 0,77 & 0,98 \\
\hline$r^{2}$ & 1,00 & 0,99 & 1,00 & 0,99 & 1,00 & 0,99 \\
\hline$\Delta \mathrm{q}_{\mathrm{e}}(\%)$ & 3,16 & 6,24 & 16,79 & 11,76 & 1,86 & 8,67 \\
\hline
\end{tabular}

Los valores obtenidos con los coeficientes de correlación cercanos a 1 indican que los modelos de Langmuir, Freundlich y Redlich Peterson son los que se correlacionan mejor con los datos experimentales. Dada la similitud observada entre los coeficientes de correlación para los modelos Langmuir (sistema homogéneo) y Freundlich (sistema heterogéneo), se utilizó el parámetro "G” del modelo de Redlich-Peterson para poder determinar la influencia de cada uno de esos modelos en el proceso de adsorción. 
Tabla 3. Parámetros de las isotermas obtenidas de la adsorción de metales pesados empleando los carbones activados AG0,75_600 y AG1_600.

\begin{tabular}{|c|c|c|c|c|c|c|}
\hline \multirow[b]{2}{*}{ Muestra } & \multicolumn{2}{|c|}{$\mathrm{Pb}(\mathrm{pH} \mathrm{4})$} & \multicolumn{2}{|c|}{$\mathrm{Cr}(\mathrm{pH} \mathrm{5})$} & \multicolumn{2}{|c|}{ Cd (pH 5) } \\
\hline & 0,75 & 1 & 0.75 & 1 & 0,75 & 1 \\
\hline \multicolumn{7}{|l|}{ Freundlich } \\
\hline $\mathrm{K}_{\mathrm{F}}$ & 36,76 & 25,64 & 9,14 & 7,28 & 12,03 & 7,69 \\
\hline $1 / \mathrm{n}$ & 0,17 & 0,24 & 0,18 & 0,20 & 0,19 & 0,25 \\
\hline$r^{2}$ & 0,98 & 0,96 & 0,96 & 0,99 & 0,95 & 0,92 \\
\hline$\Delta \mathrm{q}_{\mathrm{e}}(\%)$ & 4,76 & 7,26 & 7,74 & 2,15 & 20,05 & 8,49 \\
\hline \multicolumn{7}{|l|}{ Langmuir } \\
\hline Qm & 73,34 & 71,79 & 18,75 & 17,83 & 26,33 & 17,41 \\
\hline $\mathrm{K}_{\mathrm{L}}$ & 0,50 & 0,23 & 0,64 & 0,20 & 0,46 & 1,63 \\
\hline$r^{2}$ & 0,98 & 0,97 & 1,00 & 0,99 & 0,99 & 1,00 \\
\hline$\Delta \mathrm{q}_{\mathrm{e}}(\%)$ & 33,76 & 21,71 & 23,59 & 16,51 & 25,52 & 28,73 \\
\hline \multicolumn{7}{|l|}{ Temkin } \\
\hline B & 0,30 & 0,22 & 1,09 & 1,01 & 0,77 & 1,10 \\
\hline $\mathrm{A}_{\mathrm{T}}$ & 113,24 & 7,99 & 72,10 & 14,41 & 53,43 & 75,25 \\
\hline$r^{2}$ & 0,93 & 0,92 & 0,99 & 0,99 & 0,98 & 0,99 \\
\hline$\Delta \mathrm{q}_{\mathrm{e}}(\%)$ & 8,66 & 8,90 & 4,89 & 3,01 & 9,04 & 49,08 \\
\hline \multicolumn{7}{|c|}{ Redlich-Peterson } \\
\hline $\mathrm{G}$ & 0,84 & 0,77 & 0,97 & 0,86 & 0,86 & 0,89 \\
\hline$A_{R P}$ & 1500 & 250 & 30 & 20 & 90 & 85 \\
\hline $\mathrm{B}_{\mathrm{RP}}$ & 38,39 & 8,91 & 1,90 & 2,13 & 6,06 & 7,25 \\
\hline$r^{2}$ & 1,00 & 0,99 & 1,00 & 1,00 & 1,00 & 0,99 \\
\hline$\Delta \mathrm{q}_{\mathrm{e}}(\%)$ & 5,51 & 7,04 & 3,16 & 2,18 & 10,90 & 5,24 \\
\hline
\end{tabular}

De acuerdo a los resultados obtenidos, los valores de "G" cercanos a 1 indican que en general, la adsorción es predominantemente del tipo Langmuir, lo cual indica que el proceso de adsorción se lleva a cabo en centros activados homogéneos y equivalentes en energía en toda la superficie del CA. En forma particular, para el caso del plomo, se encontró que los valores de "G" eran más bajos en comparación con los otros metales, lo que indicaría la formación de un sistema de adsorción más heterogéneo.

En el caso del ion de cromo, que es un ácido duro, se observó que en las muestras con valores de "G" cercanos a 1, la retención del cromo es mayor y esto se lleva a cabo en un sistema más homogéneo. Por otro lado, también se observó que los valores del parámetro $\mathrm{B}_{\mathrm{RP}}$ del modelo Redlich-Peterson (relacionado con la energía de adsorción) son más altos cuando el pH de la solución metálica es más ácido ( $\mathrm{pH} 2)$.

Según los valores de la desviación estándar normalizado, el modelo que mejor ajuste presentó, fue el de Redlich Peterson. En general, se podría decir que la adsorción a $\mathrm{pH} 2$ fue principalmente del tipo Langmuir para el caso del cadmio, sin embargo, para el caso del plomo 
y cromo fue Freundlich (con excepción de AG1_600 en contacto con cromo). Al aumentar el pH se observó una curva de adsorción con mayor similitud al modelo de Freundlich en todos los casos.

Finalmente, la máxima capacidad de adsorción se logró con el carbón AG0,75_600 con soluciones de un pH de 4 y 5 en el siguiente orden descendente: plomo (74,8 $\left.\mathrm{mg} \mathrm{g}^{-1}\right)$, cadmio $\left(26,5 \mathrm{mg} \mathrm{g}^{-1}\right)$, cromo $\left(18,8 \mathrm{mg} \mathrm{g}^{-1}\right)$.

\section{CONCLUSIONES}

De acuerdo con los resultados obtenidos se ha podido establecer que, en la preparación del $\mathrm{CA}$, la razón de impregnación y la temperatura de activación son los factores más influyentes en las características fisicoquímicas finales del CA

La acidez superficial del carbón, la porosidad del adsorbato $\mathbf{y}$ el pH de la solución son las variables determinantes en el proceso de adsorción de los iones $\mathrm{Cd}^{2+}, \mathrm{Cr}_{\text {(Total) }} \mathrm{y} \mathrm{Pb}^{2+}$ utilizando carbón activado.

La presencia de una mayor cantidad de centros ácidos y de un área mesoporosa más desarrollada en el carbón AG0,75_600 se atribuyó como las responsables de la mayor capacidad de adsorción presente en este carbón.

La máxima capacidad de adsorción se logró con el carbón AG0,75 600 con soluciones a un pH de 4 y 5 en el siguiente orden descendente: plomo (74,8 $\left.\mathrm{mg} \mathrm{g}^{-1}\right)$, cadmio $\left(26,5 \mathrm{mg} \mathrm{g}^{-1}\right)$, cromo $\left(18,8 \mathrm{mg} \mathrm{g}^{-1}\right)$.

Los resultados experimentales de las isotermas de adsorción tuvieron una mejor correlación con el modelo de Redlich-Peterson en todos los casos. Esto también fue confirmado por los valores normalizados de desviación estándar obtenidos.

A pH bajos $(\mathrm{pH} \leq 3)$ y en concordancia con el valor del parámetro $\mathrm{G}$ (cercano a 1 ), se puede establecer que el proceso de adsorción sigue un comportamiento descrito por el modelo de Langmuir, sin embargo, al incrementarse el pH de la solución, la adsorción ocurre en sitios más heterogéneos siguiendo el modelo de Freundlich.

\section{AGRADECIMIENTOS}

Los autores agradecen el apoyo financiero brindado por la Dirección de Gestión de la Investigación de la Pontificia Universidad Católica del Perú (DGI-PUCP) a través del Proyecto Interdisciplinario DGI 2010-0099.

\section{REFERENCIAS}

1. Agency for toxic substances and disease registry, ATSDR. The Priority List of Hazardous Substances That Will Be the Subject of Toxicological Profiles. Disponible en: http://www.atsdr.cdc.gov/SPL/index.html. Acceso: 22 Julio 2013.

2. Ministerio del ambiente. Decreto Supremo $N^{\circ}$ 002-2008-MINAM. Estándares Nacionales de Calidad de Ambiental para Agua. Categoría 1: Poblacional y recreacional. Estándares. Lima: El Peruano, 2008.

3. Obregón-Valencia D. Estudio comparativo de la capacidad de adsorción de cadmio utilizando carbones activados preparados a partir de semillas de aguaje y de aceituna. Tesis (Licenciatura en Química) - Facultad de Ciencias e Ingeniería, Pontificia Universidad Católica del Perú, Lima, 2011.

4. Pinedo A.; Obregón-Valencia D.; Sun-Kou M.R. Estudio de la capacidad de adsorción de cadmio y cromo totales utilizando carbones activados preparados a partir de semillas de 
aguaje. En: II WORKSHOP ON ADSORPTION, CATALYSIS AND POROUS MATERIALS. Resúmenes. Bogotá, Universidad de los Andes. 2013; 103-104.

5. Puziy A.; Poddubnaya O.; Socha R.; Gurgul J.; Wisniewski M. XPS and NMR studies of phosphoric acid activated carbons. Carbon, 2008; 46:2113-2123.

6. Puziy A.; Poddubnaya O.; Martínez-Alonso A.; Suárez-García F.; Tascón J. Surface chemistry of phosphorous-containing carbons of lignocellulosic origin. Carbon, 2005; 43: 2857-2868.

7. Giles, C.; Smith D. A general treatment and classification of the solute adsorption isotherm: I. Theoritical. J Colloid Interf Sci, 1974; 47: 755-765.

8. Rao M.M.; Ramana D.K.; Seshaiah K.; Wang M.C, Chien S.W. Removal of some metal ions by activated carbon prepared from Phaseolus aureus hulls. J Hazard Mater, 2009; 166: 1006-1013.

9. Wilson K.; Yang H.; Seo C.W.; Marshall W.E. Select metal adsorption by activated carbon made from peanut shells. Bioresour Technol, 2006; 97: 2266-2270.

10. El-Hendawy A.N. The role of surface chemistry and solution $\mathrm{pH}$ on the removal of $\mathrm{Pb}^{2+}$ and $\mathrm{Cd}^{2+}$ ions via effective adsorbents from low-cost biomass. J Hazard Mater, 2009; 167:260-267.

11. Pandey A.K.; Pandey S.D.; Misra V. Stability constants of metal humic acid complexes and Its Role in Environmental Detoxification. Ecotoxicol Environ Saf, 2002; 47: 195200.

12. Alfarra A.; Frackowiak, E.; Béguin, F. The HSAB concept as a means to interpret the adsorption of metal ions onto activated carbons. Appl Surf Sci, 2004; 228,: 84-92. 